\title{
Spatial Configuration and Users' Behavior in Co-working Spaces
}

\author{
How spatial elements cue behaviors \\ Eric Prince Ondia ${ }^{1}$, Sirimas Hengrasmee ${ }^{2}$, and Sant Chansomsak ${ }^{3}$ \\ ${ }^{1}$ Department of Architecture, Naresuan University, Phitsanulok, Thailand \\ erico59@email.nu.ac.th \\ ${ }^{2}$ Department of Architecture, Naresuan University, Phitsanulok, Thailand \\ sirimash@nu.ac.th \\ ${ }^{3}$ Department of Architecture, Naresuan University, Phitsanulok, Thailand \\ santc@nu.ac.th
}

\begin{abstract}
This paper aims to examine whether there is a direct relationship between spatial configuration and users' behaviors in co-working spaces, and if so, how this environment and behavior relationship impacts their working process. The study employed ethnographic qualitative strategy as the general method of inquiry and used visual documentation, direct observations, and behavioral mapping as methods of data collection in two case studies. Analysis of the findings demonstrates that design elements such as barriers and fields are powerful tools for influencing and guiding users' behavior within coworking spaces. The findings provide a deeper understanding of the relationship between design and behavioral patterns in co-working spaces. The research insights in this study may inform architects, policymakers and facility managers in making conscious decisions on the design of co-working spaces that are more meaningful to the users.
\end{abstract}

Keywords: Co-working space, spatial design, spatial behavior.

\section{INTRODUCTION}

In the present knowledge economy, workers are becoming more and more mobile in work locations due to improved telecommunications [1]. These workers enjoy flexibility in work locations because they can work from home or any other place as opposed to going to an office. This growing phenomenon has prompted the rise of new alternative working environments named 'co-working spaces' to support more mobile and flexible work styles [2]. Spreitzer, Bacevice and Garrett [3] define co-working spaces as "membership-based workspaces where diverse groups of freelancers, remote workers, and other independent professionals work together in a shared, communal setting". Unlike traditional offices that have formal structures involving managers and employees, whereby employees may have well-defined work roles, coworking space members are not constrained by any organizational norms in terms of expected behavior. The users are independent with regards to their behavior. Co-working spaces provide users with a space to work independently, and at the same time, they give users opportunities for collaboration and interaction with like-minded people. This kind of arrangement seems to reflect the lifestyle needs of the emerging workforce dominated by millennials. To date, studies related to the social, psychological, and physical needs associated with co-working environments are limited. This study aims to shed light on the following question: Does spatial configuration of co-working spaces impact users' behaviors, and, if so, how? 
Previous studies on work environments agree that spatial properties have effects on the way workers behave [4-6]. This paper endeavors to expand on this contention and apply existing environment-behavior theory to establish the relationship between design and behavior in coworking spaces. Users' behaviors are measured based on the four work modes of a workplace [7]-focus, collaborate, socialize and learn. To describe the spatial characteristics of coworking spaces, the study mobilized Zeisel's [8] categories of design features related to spatial behaviors-barriers and fields. Specific barriers investigated in this study include walls, screens, and objects. Fields examined include size and orientation.

\section{THEORY AND LITERATURE REVIEW}

\subsection{Theoretical framework}

This study builds on the theory of behavior setting [9], the theory of environmental affordance [10], and the theory of proxemics of space [11]. The three theories emphasize the mutuality of people and their environment and have been recognized as foundations for environmentbehavior research [12].

\subsubsection{Behavior setting}

The collective actions of people with respect to the physical environment is what defines a behavior setting. The idea of behavior setting was introduced by Barker [9]. A behavior setting involves a particular layout of the environment, a recurrent activity, and a synomorphy or a congruent relationship between the two $[9,12]$. The greater the congruent relationship, the better the behavior setting is able to afford people's behaviors. The meaning of the setting, in terms of what type of place it is and what kind of behaviors are appropriate in it, depends on the perceived congruent relationship between the features of the setting and behavior occurring in it. The idea of behavior setting is used in this investigation by dividing the co-working environment into various spaces, for example, workstations, informal seating spaces, and breakout spaces, rather than regarding the co-working space as a general context for behavior. Connecting the type of space and the level of activity is necessary in order to develop a better comprehension of the effect of spatial configuration on users' behavior.

\subsubsection{Environmental affordance}

Affordances are the perceived properties of the physical environment that enables it to be ued for some activity [10]. Unlike behavior settings that involve the collective actions of individuals, the idea of affordance involves the actions of specific perceivers. Therefore, the affordance of an object or setting may change with the needs, the cultural and individual backgrounds of the various perceivers [12]. The concept enables researchers to gain a better understanding of how spatial elements facilitate users' behaviors. The idea of affordance is applied in this investigation to understand the commonalities and variance between various spaces by depicting the physical characteristics of spaces that offer particular behavioral reactions. For instance, nooks or alcoves for having private discussions.

\subsubsection{Proxemics of space}

The term Proxemics was authored by Edward T. Hall to mean the study of the means by which individuals and groups communicate through their utilization of space [11]. 
Hall identified three categories of spaces related to spatial behaviors: fixed-feature, semifixed-feature, and informal spaces [11]. Fixed-features are the permanent spatial components that articulate space, for example, walls, doors, and windows. Semi-fixed-features are the movable spatial components that define space, for example, furniture and movable partitions. Hall classified Semi-fixed-feature spaces as socio-petal and socio-fugal. Socio-petal spaces are those that have a tendency to bring individuals together whereas, socio-fugal spaces are those that have a tendency to keep individuals apart. Informal space refers to the interpersonal distances individuals unwittingly keep during interpersonal relations [11].

With regard to informal space, Hall identified four distances or zones in which people interact contingent upon the kind of relationship they share with others. These distances are named intimate, personal, social and public distances [11]. Intimate distance (0-0.45 meters) is that zone immediately surrounding a person's body. This zone is the most private and it is reserved for physical and emotional interactions. Personal distance $(0.45-1.2$ meters $)$ is that zone within which a person only permits close friends or fellow workmates with whom personal conversation is necessary. Social distance (1.20-3.60 meters) is that zone within which a person seeks to make absolutely social contacts on a temporary basis. It is utilized for conversing with somebody who is an outsider or not well known. Public distance $(3.60-7.50+$ meters $)$ is that zone within which a person does not seek direct contact with others. It is utilized for public speaking, such as addressing a crowd.

The understanding of the above mentioned three types of spaces (fixed-feature, semi-fixedfeature, and informal spaces) is necessary to peruse the behavior of the participants in co-working environments.

\subsection{Elements of the physical environment associated with spatial behaviors inwork environments}

Zeisel [8] categorizes properties of the physical environment associated with spatial behaviors as barriers and fields. Barriers are the physical elements in work environment that keep people apart or joining them together, physically and symbolically [8]. Barriers include walls, screens, objects, and symbols. Having walls in a space separates people and their absence joins people. Brill, Margulis, Konar and BOSTI [13] demonstrates that individuals regulate their interpersonal accessibility most consistently through physical enclosures of the workspace, such as walls and partitions. Screens, which include doors, windows and glass panels are more selective in separating and joining people than walls. For example, glass provides tactile separation between people in a place yet it allows visual connection between them [8]. Objects placed in a space such as a furniture may either facilitate separation or connection for the occupants in a space [8]. Symbols which include floor level changes or color changes in a room may separate or join people perceptually. For instance, people may consider two places with varying floor levels as separate places [8].

Fields are the physical elements in work environments that perceptually separate or join people together by altering the physical context in which perceptual relationships occur [8]. Fields include size, shape, orientation and environmental conditions. Size of a setting can either give an opportunity for people to adjust their interpersonal distance or limit their options for separation. For instance, a large space allows people to separate themselves from others while a small space restricts them from separating from others [8]. The shape of a setting can perceptually separate or join people in a place. For instance, corners in a square shape can easily be viewed as separate from each other, which is not the case with round shapes that join people [8]. Positioning in space, or orientation joins or separates people perceptually through functional distance. For instance, two places oriented in such a way that people using them can easily 
encounter each other are considered functionally closure and two places oriented in such a way that people using them cannot easily encounter one another are considered functionally distant [8]. Environmental conditions, which includes loudness, light intensity and airflow perceptually separate or join people by facilitating or limiting their ability to see, smell and hear other people and their activities in a place [8]. As an environmental condition, lighting systems that give higher light levels on the primary work surfaces, and decrease general surrounding light levels make a varyingly lit workspace [14]. This perceptually separates the primary work surface from other areas in the same space.

\subsection{Knowledge workplace activities: Four work modes}

Research shows that knowledge work is typically accomplished in four different modes, namely, focus, collaborate, socialize and learn [7]. Focus mode involves activities in the workplace that require concentration [7]. Heerwagen, Kampschroer, Powell and Loftness [15] point out that there are two basic needs of knowledge workers: Time to work alone to think, analyze and reflect; and time to interact with others so that ideas can be generated and evaluated. It is therefore important for co-working spaces to ensure that users can focus and work individually when needed with minimal distractions. Collaborate mode involves workplace activities whereby an individual works with another individual or group to accomplish a shared objective [7]. Collaborate mode is associated with support for user proximity and visual contact for "people to interact frequently and build the relationships that help them share information, think creatively and reach more innovative solutions" [7]. Socialize mode involves the interactions in the workplace that create common bonds and productive relationships among the users [7]. Socialization helps create a sense of community through building pathways of information sharing among occupants of the workplace [7]. Learn work mode "refers to the activity of acquiring new knowledge of a subject or skill through education or experience" [7]. This mode of working involves creating and building new knowledge, which might be gained through seminars and presentations.

The best-performing work environments bolster every one of the four work modes [7]. This investigation places emphasis on understanding how the design of co-working spaces supports or inhibits these four work modes.

\section{METHOD}

To achieve the aim of this research, the study used ethnographic qualitative research strategy as the general method of inquiry and involved case studies in two co-working spaces located in Chiang Mai, Thailand. The two case studies include Punspace Nimman and Punspace Tha Phae Gate. Both sites comprise of a wide range of entrepreneurs and start-ups. The majority of the community works in the tech and IT industry, with age range between twenty to thirty-five years. The methods of data collection involved visual documentation, direct observations, and behavioral mapping. Visual documentation involved recording the physical aspects of the site through photographs and drawings. Direct observations made it possible to note the activities people were carrying out, how they were carrying out those activities, and their attitudes [1618]. The goal was to discover the meaning of the space to the participants. Behavioral mapping was used for recording the users' activities and their locations on the plan of the site [19]. The goal was to link design features to specific activity types in co-working spaces.

Each co-working space was studied for 24 hours on a variety of days and time intervals from 4th December 2017 to 14th December 2017. Each study began with visual documentation. After visual documentation, direct observations and behavioral mapping were carried out hand in hand. 
During this process, the behaviors taking place at each research site were recorded on a copy of the floor plan of the site. In addition, field notes were taken to describe detailed behaviors. Every behavior observed was denoted with a letter on the floor plan used for behavioral mapping, which matched a similar letter in the field notes for recording direct observations. This method was helpful by allowing the field notes and floor plans to be studied further after the observation sessions. Data obtained through behavioral mapping was correlated with the data obtained through direct observations to achieve a meaningful understanding of users' behavior in the co-working space because behavioral mapping alone does not reveal a person's emotional engagement with space. After considering the nature of each co-working space separately, the emerging behavioral trends in the two case studies were compared to determine the commonalities and variance in users' behaviors. These comparisons made it possible to draw conclusions about the association between design features and users behavior in co-working spaces.

\section{ANALYSIS AND COMPARISON}

For the two co-working spaces, behavioral observations were distributed across seven types of spaces: workstation, informal seating area, breakout space, kitchen/coffee, reception desk, printer/copy, and circulation route.

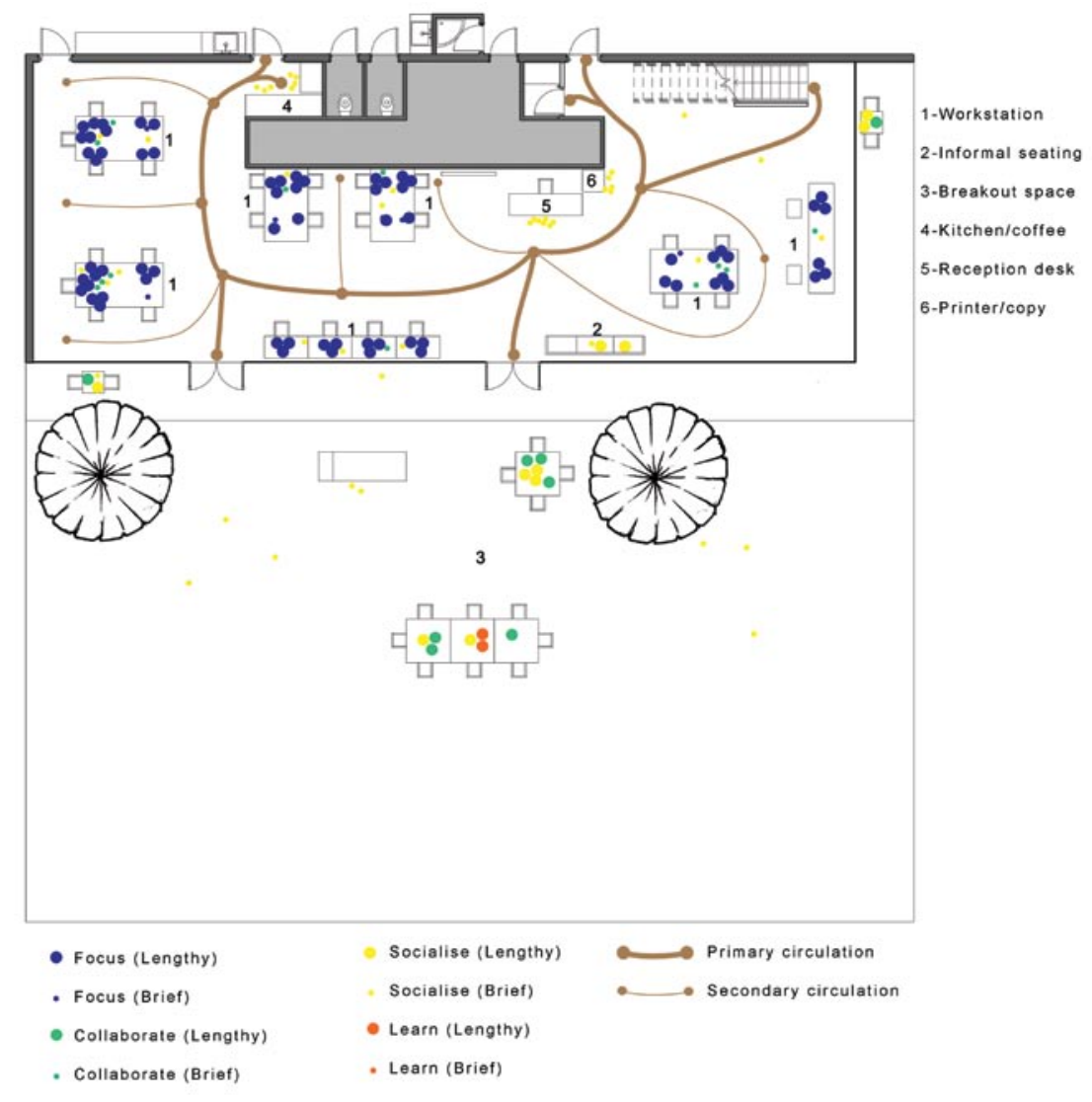

Figure 1. Behavioral map of users' activities at Punspace Nimman. 


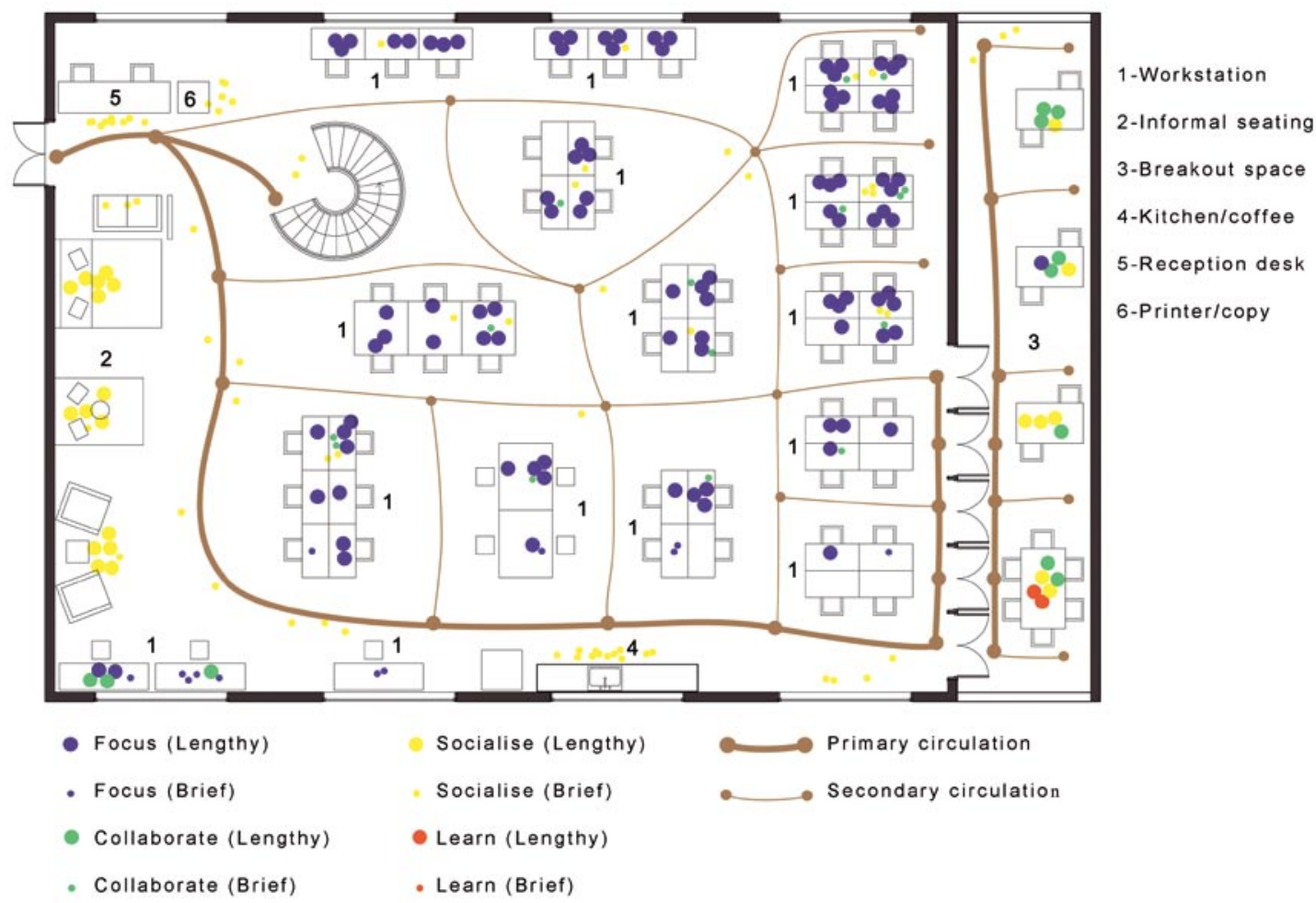

Figure 2. Behavioral map of users' activities at Punspace Tha Phae Gate

From the behavioral maps, spaces with minimal and highest levels of various behaviors were identified to determine the relationship between spatial components and patterns of behaviors. This information made it possible to generate a frequency table of users' activities in the two research sites.

\begin{tabular}{|c|c|c|c|c|c|c|c|c|}
\hline \multirow{3}{*}{$\begin{array}{l}\text { Behavior } \\
\text { setting }\end{array}$} & \multicolumn{8}{|c|}{ Activities } \\
\hline & \multicolumn{2}{|l|}{ Focus } & \multicolumn{2}{|c|}{ Collaborate } & \multicolumn{2}{|l|}{ Socialise } & \multicolumn{2}{|l|}{ Learn } \\
\hline & $\begin{array}{l}\text { Frequency } \\
\text { of activity }\end{array}$ & $\%$ & $\begin{array}{l}\text { Frequency } \\
\text { of activity }\end{array}$ & $\%$ & $\begin{array}{l}\text { Frequency } \\
\text { of activity }\end{array}$ & $\%$ & $\begin{array}{l}\text { Frequency } \\
\text { of activity }\end{array}$ & $\%$ \\
\hline Workstation & 74 & 100 & 12 & 60 & 14 & 25.5 & - & - \\
\hline Informal seating & - & - & - & - & 3 & 5.5 & - & - \\
\hline Breakout space & - & - & 8 & 40 & 17 & 30.9 & 2 & 100 \\
\hline Kitchen/coffee & - & - & - & - & 7 & 12.7 & - & - \\
\hline Reception desk & - & - & - & - & 7 & 12.7 & - & - \\
\hline $\begin{array}{l}\text { Circulation } \\
\text { routes }\end{array}$ & - & - & - & - & 2 & 3.6 & - & - \\
\hline Printer/copy & - & - & - & - & 5 & 9.1 & - & - \\
\hline Total & 74 & 100 & 20 & 100 & 55 & 100 & 2 & 100 \\
\hline
\end{tabular}

Table 1. Frequency of users' activities in various behavior settings at Punspace Nimman 


\begin{tabular}{|c|c|c|c|c|c|c|c|c|}
\hline \multirow{3}{*}{$\begin{array}{l}\text { Behavior } \\
\text { setting }\end{array}$} & \multicolumn{8}{|c|}{ Activities } \\
\hline & \multicolumn{2}{|l|}{ Focus } & \multicolumn{2}{|c|}{ Collaborate } & \multicolumn{2}{|l|}{ Socialise } & \multicolumn{2}{|l|}{ Learn } \\
\hline & $\begin{array}{l}\text { Frequency } \\
\text { of activity }\end{array}$ & $\%$ & $\begin{array}{l}\text { Frequency } \\
\text { of activity }\end{array}$ & $\%$ & $\begin{array}{l}\text { Frequency } \\
\text { of activity }\end{array}$ & $\%$ & $\begin{array}{l}\text { Frequency } \\
\text { of activity }\end{array}$ & $\%$ \\
\hline Workstation & 108 & 99 & 18 & 69.2 & 17 & 18.7 & - & - \\
\hline Informal seating & - & - & - & - & 17 & 18.7 & - & - \\
\hline Breakout space & 1 & 1 & 8 & 30.8 & 8 & 8.8 & 2 & 100 \\
\hline Kitchen/coffee & - & - & - & - & 12 & 13.2 & - & - \\
\hline Reception desk & - & - & - & - & 12 & 13.2 & - & - \\
\hline $\begin{array}{l}\text { Circulation } \\
\text { routes }\end{array}$ & - & - & - & - & 19 & 20.8 & - & - \\
\hline Printer/copy & - & - & - & - & 6 & 6.6 & - & - \\
\hline Total & 109 & 100 & 26 & 100 & 91 & 100 & 2 & 100 \\
\hline
\end{tabular}

Table 2. Frequency of users' activities in various behavior settings at Punspace Tha Phae Gate

Information obtained through behavioral mapping was correlated with the data acquired through direct observations to gain a significant comprehension of the relationship between design features and behavioral patterns. Below, each activity type is discussed with regards to how it related to the spatial design elements in the two co-working spaces investigated.

\subsection{Focus}

In the two case studies, focused work seemed to primarily take place at the workstations. The preference of the workstations for concentrating could be as a result of the privacy offered by the workstations in comparison to social spaces such as breakout spaces and informal seating areas. Focus mode in the two co-working spaces seemed to be influenced by certain barriers and fields investigated in this study as discussed below.

\subsubsection{Barriers}

\subsubsection{Walls}

Having a solid wall at the back or on one side of the workstation appeared to be associated with support for concentrated work at Punspace Nimman and Punspace Tha Phae Gate. Users expressed their preference for seats positioned adjacent to solid walls while concentrating on their task. These seats were positioned with the wall either at the back or on the side. This behavior could be attributed to the protection offered by walls from spatial intrusion on at least one side, thereby giving users some control of interpersonal accessibility.

Having a floor-to-ceiling wall separating the open workstations from the breakout space seemed to be related with facilitation of concentration among the users at Punspace Tha Phae Gate. Unlike Punspace Nimman, whereby a glass wall was used to separate the open workstations from the breakout space, at Punspace Tha Phae Gate, the two spaces were acoustically separated by a solid wall. The wall minimized acoustic distractions at the open workstations by blocking potential auditory distractions caused by verbal communications in the breakout space. This allowed users to focus on their work at the workstations without being distracted by people having verbal communications in the breakout space. 


\subsubsection{Screens}

Having a window or glass wall in front of the workstation that allows an outside view appeared to be associated with support for concentration among the users in the two research sites. Unlike seats positioned adjacent to solid walls that have the wall at the back or on the side, these seats were positioned facing the window or glass wall in order to have a visual connection to the outside space. The reason these seats were favored by the users during concentration appeared to be because inside activities were cut off from their view while those outside may not have been as visually distracting because they were out of their immediate reach tactilely and acoustically

Having a glass wall acoustically separating the open workstations from the outdoor breakout space seemed to facilitate concentration among the users at Punspace Nimman. Unlike Punspace Tha Phae Gate, whereby the open workstations were separated from the breakout space by a solid wall, at Punspace Nimman, glass walls were utilized to acoustically separate the two spaces. This acoustic separation allowed users to focus on their work at the workstations without being distracted by verbal communications in the outdoor breakout space. Besides providing acoustic privacy, the glass walls allowed visual between the workstations and the breakout space.

\subsubsection{Objects}

Having a spacious work surface with enough room to place personal belongings appeared to be associated with support for concentration among the occupants at Punspace Nimman and Punspace Tha Phae Gate. Users in the two co-working spaces had a tendency to mark their workspace with personal items such as coffee cups and notebooks to define their territory during moments of focused work. This behavior shows an effort by the users to regulate their privacy through territorial behavior while concentrating.

\subsubsection{Fields}

\subsubsection{Orientation}

Greater distance between workstations and primary circulation routes appeared to be associated with support for focused work in the two research sites. Occupants at Punspace Nimman and Punspace Tha Phae Gate preferred to position themselves at a greater distance from primary circulation routes while carrying out focused work. This behavior appeared to be linked to the need for visual and acoustic privacy during concentration. Primary circulation routes appeared to be points of visual and acoustic distractions, especially during peak hours when many people were passing by. In many occasions, users concentrating on their work in close proximity to primary circulation routes looked up when other people walked nearby, seeming interrupted or distracted. Distraction from these points required users to continuously refocus from breaking their concentration.

Greater workstation-to-doorway distance seemed to be associated with support for concentration among the users at Punspace Nimman and Punspace Tha Phae Gate. Doorways appeared to be points of visual and acoustic distractions in the two research sites. Distraction from people opening and closing the door continuously caused individuals concentrating on their work nearby to glance whenever someone opened or closed the door, seeming distracted.

A greater workstation distance from shared service and amenity spaces appeared to be an important spatial element for carrying out focused work in the two case studies. 
Shared service and amenity spaces like kitchen/coffee area and printer/copy area seemed to add to the acoustical distractions users encounter in the co-working space. Much as these spaces were critical for casual social interactions by providing opportunities for unscheduled encounters between the users, they likewise appeared to create auditory distractions to those focusing on their work nearby. The location of these spaces seemed to be favorable at certain distances from the workstations to ensure lower levels of visual and auditory distractions to people concentrating at the workstations.

Adequate proximity between adjacent workstations seemed to be associated with support for concentrated work in the two research sites. Users at Punspace Nimman and Punspace Tha Phae Gate preferred to keep a certain distance between themselves and others while concentrating on their work. On individual workstations arranged side by side (Socio-fugal), the majority of the users preferred to sit at least two seats away from others. While on the shared workstations arranged to facilitate eye contact (Socio-petal), the majority of the users kept even a further interpersonal distance by choosing to sit diagonally across the workstations. This behavior appeared to be linked to the need for personal space while concentrating. Users attempted to gain privacy by using these spatial distances.

In the two research sites, socio-fugal desk arrangements seemed to be associated with support for concentrated work. During seat selection for concentrated work, socio-fugal desks were often selected before socio-petal desks. This appeared to be because socio-petal arrangements force social intimacy that might have been intimidating to the users while concentrating on their work.

\subsection{Collaborate}

In the two research sites, collaborative work mostly occurred at the workstations and in the breakout spaces. While the highest percentage of the collaborative work in the two co-working spaces occurred at the workstations, these activities were not for an extended period of time. Extensive collaborations primarily occurred in the breakout spaces. It seemed the open layout of these spaces might have prevented high levels of verbal communication at the workstations because users might not want others to overhear their discussions or they might not want to cause auditory distractions to others who were concentrating on their work at the workstations close by. Collaboration in the two co-working spaces appeared to be impacted by certain barriers and fields investigated in this study as discussed below.

\subsubsection{Barriers}

\subsubsection{Walls}

Having a floor-to-ceiling wall separating the open workstations and the breakout space seemed to be associated with facilitation of collaborative work at Punspace Tha Phae Gate. Unlike Punspace Nimman whereby the workstations were separated from the breakout space by a glass wall, a solid wall was used to separate the two spaces at Punspace Tha Phae Gate. The solid wall provided visual and acoustic privacy between the two spaces and therefore appeared to facilitate collaborative work by allowing users to freely have verbal communications during collaborative work in the breakout space without feeling as though they were causing auditory distractions to those carrying out focused work at the workstations. 


\subsubsection{Screens}

Having a glass wall acoustically separating the open workstations from the breakout space appeared to be associated with support for collaborative work among the occupants at Punspace Nimman. Unlike Punspace Tha Phae Gate, whereby the open workstations were separated from the breakout space by a solid wall, at Punspace Nimman, a glass wall is used to separate the two spaces. The glass wall provided acoustic privacy between the two spaces and therefore seemed to facilitate collaborative work by allowing users to have verbal communications in the outdoor breakout space during moments of collaboration without feeling as though they were distracting others who might be concentrating on their work at the workstations.

\subsubsection{Objects}

Flexible and adaptable furniture appeared to be associated with support for collaborative work among the occupants at Punspace Nimman and Punspace Tha Phae Gate. The ability to pull two tables together allowed various users to join or separate seats depending on their group size and their privacy needs. This made it possible for groups to set up temporary territories during collaborative work. Flexible furniture also allowed individuals in a group to regulate their interpersonal distances during discussions and therefore giving them control of their personal space.

Spacious work surfaces seemed to be associated with support for collaborative work among the occupants at Punspace Nimman and Punspace Tha Phae Gate. Collaboration in the two research sites appeared to be facilitated when the location offered a spacious work surface for the desired group size. Spacious work surfaces that enable individuals in a group to occasionally move apart or closure to their counterparts seemed to be critical during collaborative work. They allowed users to have control of their interpersonal contact during collaborative work. Small work surfaces tend to restrict users from adjusting their interpersonal distance, thus limiting their capacity to control their personal space. Users in the two co-working spaces often joined tables to create larger work surfaces to facilitate collaborative work.

\subsubsection{Fields}

\subsubsection{Orientation}

Socio-petal seat arrangements seemed to be associated with support for collaboration among the users at Punspace Nimman and Punspace Tha Phae Gate. Users in the two co-working spaces expressed their preference for socio-petal seats while carrying out collaborative work. This preference seemed to be linked to the need for eye contact for communication. Socio-fugal seats discourage eye contact which seemed to be a hindrance for collaborative work among the users in the two research sites.

Greater workstation distance to primary circulation routes seemed to be associated with support for collaborative work in the two research sites. People's movements along primary circulation routes appeared to cause a visual and acoustic distraction for users during moments of collaboration. Users collaborating nearby primary circulation routes often looked up seeming interrupted when other users passed by their workstations. 
A greater distance between workstations and doorways seemed to be an important spatial lement for collaborative activities at Punspace Nimman and Punspace Tha Phae Gate. Auditory and visual distractions from people opening and closing the door continuously caused team members to look up seeming distracted during collaborative work.

A greater distance between workstations and shared service and amenity spaces seemed to be associated with support for collaborative behaviors among the users in the two research sites. Shared service and amenity spaces seemed to provide opportunities for unscheduled encounters between the users for casual social interactions, however, they likewise appeared to add to the auditory distractions users encounter during moments of collaboration.

An adequate distance between adjacent workstations appeared to be associated with facilitation of collaborative behaviors among the occupants at Punspace Nimman and Punspace Tha Phae Gate. Groups who visited the two co-working space with the intention of collaborating had a tendency to avoid workstations placed excessively close to each other. This appeared to be because the users might not want others to overhear their conversations during collaborations. In most cases, users would move their workstations away from others when they felt these workstations were excessively close to each other.

\subsubsection{Size}

A spacious workspace that permits flexible movement seemed to be related with support for collaborative work at Punspace Nimman at Punspace Tha Phae Gate. The limited workspaces size of Punspace Nimman prevented small groups from distancing themselves from others for having private discussions at the open workstations. At the point when users desired to be separated from everyone else for having confidential discussions, they would move to the outside breakout space. The plenteous space for adaptable movement in the breakout space seemed to encourage collaboration among the users by enabling small groups to effectively distance themselves from others when they required privacy for collaboration. At Punspace Tha Phae gate, the fairly large workspace appeared to facilitate collaborative work among the occupants by allowing small groups to easily distance themselves from others when they needed privacy for collaborating. When there were few users in the co-working space, small groups could easily distance themselves from others to have private collaborations at the workstations without having to leave the room. When there were many users in the co-working space, it was not possible for the users to distance themselves from others and therefore they relocated to the breakout space in order to have private collaborations. This behavior could be linked to the feeling of crowding users experience in limited spaces without possibilities of separating from others.

\subsection{Socialize}

In the two research sites, there seemed to be some variations in the spaces where high levels of social interaction occurred. The breakout space at Punspace Nimman carried a significantly higher percentage of social interactions in comparison to the breakout space at Punspace Tha Phae Gate. Similarly, the informal seating area at Punspace Tha Phae Gate carried a significantly higher percentage of social interactions compared to the informal seating area at Punspace Nimman. These variations appeared to be associated with the size of the various spaces in the two co-working spaces. At Punspace Nimman, the breakout space provided users with abundant space that allowed them to comfortably socialize while having full control over their interpersonal contact. This seemed to have contributed to high levels of social interaction at this space. While the breakout space at Punspace Tha Phae Gate, was limited in size considering 
the number of occupants in the coworking space. This seemed to have contributed to low levels of social interactions in this space. On the other hand, the informal seating area at Punspace Nimman was limited is size. The only available seat accommodated three people at a time. This appeared to have impeded high levels of social interaction at this space. At Punspace Tha Phae Gate, the informal seating area offered abundant space with plenty of seats to choose from, therefore giving users control of their interpersonal contact during moments of social interaction. This appeared to have contributed to the high levels of social interactions in this space. Despite the fact that social interactions that occurred at the workstations carried the highest percentages in the two research sites, these interactions were not for an extended period of time. Extensive socialization generally occurred at the informal seating areas and the breakout spaces. Socializations along the circulation paths and shared service and amenity spaces were also not for an extended time, however, they added to the common bond and productive relationships needed among the users in the co-working space. Socialization in the two case studies seemed to be associated with certain barriers and fields investigated in this study as discussed below.

\subsubsection{Barriers}

\subsubsection{Walls}

Having a solid wall separating the open workstations and the breakout space appeared to be associated with support for social interactions at Punspace Tha Phae Gate. Unlike Punspace Nimman whereby the workstations are separated from the breakout space by a glass wall, at Punspace Tha Phae Gate, a solid wall is used to separate the open workstations from the breakout space. The wall provided acoustic privacy between the two spaces and allowed users to have verbal interactions in the breakout space without feeling as though they were causing auditory distractions to those concentrating on their work at the workstations.

\subsubsection{Screens}

Having a glass wall separating breakout spaces from the open workstations seemed to be associated with support for social interactions at Punspace Nimman. Unlike Punspace Tha Phae Gate whereby the workstations are separated from the breakout space by a solid wall, at Punspace Nimman, a glass wall is used to separate the open workstations from the breakout space. The glass wall provided acoustic privacy between the two spaces and allowed users to interact freely in the breakout space without feeling as though they were distracting others who might be focusing on their work at the workstations. Besides providing acoustic separation, the glass wall allowed visual connection between the workstations and the outdoor breakout space.

\subsubsection{Objects}

Flexible and adaptable furniture appeared to be associated with facilitation of social interactions at Punspace Nimman and Punspace Tha Phae Gate. Flexible furniture allowed users in the two co-working spaces to adjust their interpersonal distance during social interactions. This enabled users to have control of their personal space. Flexible furniture also made it possible for small groups of users to create temporary territories for social interactions in the two case studies, thus giving users control of their social contact during social interactions. 


\subsubsection{Fields}

\subsubsection{Size}

A spacious workspace that allows flexible movement and convenient communication appeared to be associated with support for socialization among the users at Punspace Nimman and Punspace Tha Phae Gate. At Punspace Nimman, the limited workspace size prevented small groups of users from separating themselves from others to have confidential conversations at the open workstations or the informal seating area. When users needed to be alone in small groups, they moved to the outdoor breakout space. At Punspace Tha Phae gate, the fairly large workspace size appeared to facilitate social interactions among the occupants by allowing small groups to easily distance themselves from others when they needed privacy during socialization. When there were few users in the co-working space, individuals and small groups could easily distance themselves from others to have private conversations at the workstations or the informal seating area without having to leave the room. When there were many users in the co-working space, it was not possible for the users to distance themselves from others and therefore they relocated to the breakout space in order to have longer confidential conversations.

\subsubsection{Orientation}

A socio-petal seat arrangement appeared to be associated with facilitation of socialization among the occupants in the two research sites. Users in the two co-working spaces often selected socio-petal seats or rearranged their seats to facilitate eye contact while socializing. This behavior could be linked to the need for eye contact for communication during social interactions.

\subsection{Learn}

In the two case studies, all learning activities observed occurred in the breakout spaces. No learning activities were recorded at the workstations. The reason users in the two co-working spaces never utilized the workstations for learning could be linked to the potential for auditory distractions caused by verbal communications during learning activities. Users seemed to desire a secluded space where they could freely have verbal communications without distracting others who might be concentrating on their work at the open workstations. Learning activities at Punspace Nimman and Punspace Tha Phae Gate appeared to be influenced by certain barriers and fields investigated in this study as discussed below.

\subsubsection{Barriers}

\subsubsection{Walls}

Having a solid wall separating the open workstations and the breakout space appeared to be associated with facilitation of learning activities at Punspace Tha Phae Gate. Unlike Punspace Nimman whereby the workstations are separated from the breakout space by a glass wall, at Punspace Tha Phae Gate, a solid wall was used to separate the two spaces. The wall provided acoustic privacy between the two spaces and allowed users to have verbal communications during learning activities in the breakout space without feeling as though they were distracting others who might be concentrating on their work at the workstations. 


\subsubsection{Screens}

Having a glass wall separating the open workstations and the breakout space appeared to be associated with support for learning activities at Punspace Nimman. Unlike Punspace Tha Phae Gate whereby the workstations were separated from the breakout space by a glass wall, at Punspace Nimman, a glass wall was used to separate the two spaces. The glass wall provided acoustic privacy between the two spaces and allowed users to freely have verbal communications during learning activities in the breakout space without causing auditory distractions to those concentrating on their work at the workstations. In addition to providing acoustic privacy between the two spaces, the glass wall allowed visual connection between them.

\subsubsection{Objects}

Flexible furniture appeared to be associated with support for learning activities among the users in the two research sites. Flexible furniture facilitated learning activities in such a way that it allowed users to regulate their interpersonal distances during seminars and group presentations, thus giving users control of their personal space.

Having an adequate work surfaces appeared to be associated with facilitation of learning activities at Punspace Nimman and Punspace Tha Phae Gate. Users in the two research sites often joined tables to create a spacious work surface for holding seminars and small group presentations. Spacious work surfaces seemed to allow users to easily regulate their interpersonal distances compared to small work surfaces that tend to restrict users from adjusting their interpersonal distances, thus limiting their capacity to control their personal space during learning activities.

\subsubsection{Fields}

\subsubsection{Orientation}

Socio-petal seat arrangements seemed to be associated with support for learning activities in the two research sites. Users in the two co-working spaces expressed their preference for sociopetal seats while holding small group presentations and seminars. This behavior appeared to be linked to the need for eye contact for communication among the users to proficiently carry out learning activities.

\section{DISCUSSION}

Through these comparisons, the differences in total activity that happen at various kinds of spaces or behavior settings and various forms of a similar type of spaces (open workstations, informal seating areas, breakout spaces, kitchen/coffee area, circulation routes, printer/copy area, reception desk) provided the possibility to comprehend the effect of affordances in design of co-working spaces. For instance, an informal seating area is a type of spaces normally associated with high levels of social interaction. However, the level of activity afforded can be considerably influenced by physical characteristics of the space, for example, the size. Analysis of the findings shows that design elements comprising of barriers and fields have a significant impact on users' behavior in co-working spaces. The findings validate previous research claims that barriers and fields are powerful tools for guiding people's behavior in an environment [8]. 
The consistency of the reoccurring patterns in behavior in relation to specific spatial elements in the two case studies reinforced these findings. The findings demonstrated that similar design elements were related to different activity types in co-working spaces. Some design elements that seemed to be effective for focused work also appeared to be effective for collaboration, socialization, and learning. The difference between how these elements supported a specific activity type often depended on how these elements were used. For example, the separation between the open workstations and breakout spaces appeared to facilitate collaboration, learning, and socialization in that it allowed users to have verbal communications in the breakout spaces without feeling as though they were causing auditory distractions to others who might be concentrating on their work at the workstations. That same separation, in turn, facilitated focused work by minimizing the level of auditory distractions at the open workstations by providing an acoustically separate space for the users to have verbal communications. Table 3 and 4 illustrate the association between specific spatial elements and the various activities investigated in this study. The study shows that users attach a symbolic value to physical characteristics of co-working spaces. For example, having a wall at the back or on one side appeared to contribute to the symbolic value of protection from spatial intrusion, which seemed to be critical during moments of focused work. Furthermore, the findings in the study show that due to the open plan layout of the two research sites, fields such as size and orientation seemed to be critical for the users while carrying out their works. It seemed that enclosing individual workspaces with floor-to-ceiling walls may not be necessary to solve the privacy issues associated with the open plan layout of the co-working spaces, so long as the spatial elements needed to regulate interpersonal contact are provided. For example, orientation such as having a greater workstation-to-primary circulation route distance seemed to afford focused work which is associated with higher levels of visual and acoustic privacy among the users in the two research sites. However, no one design component might fully afford users' behaviors in co-working spaces.

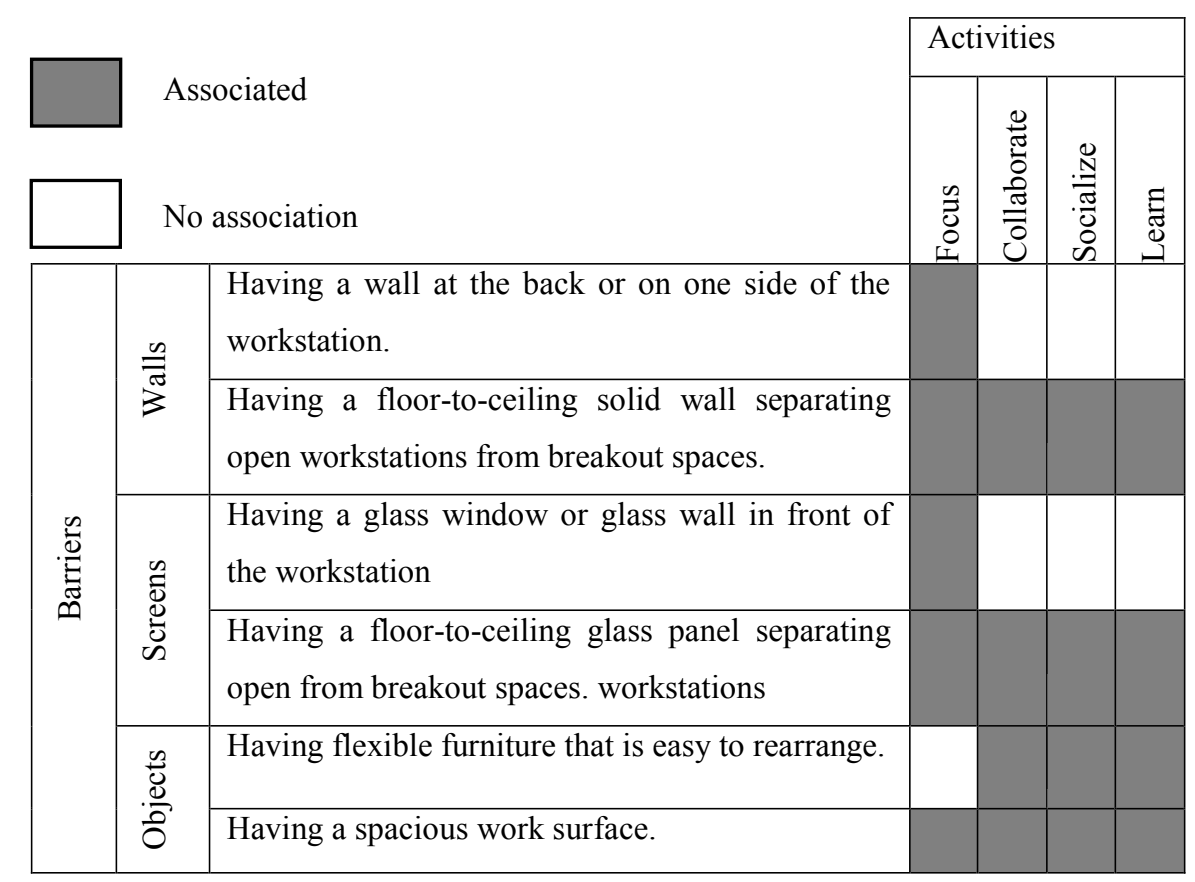

Table 3. Association between barriers and users' activities 


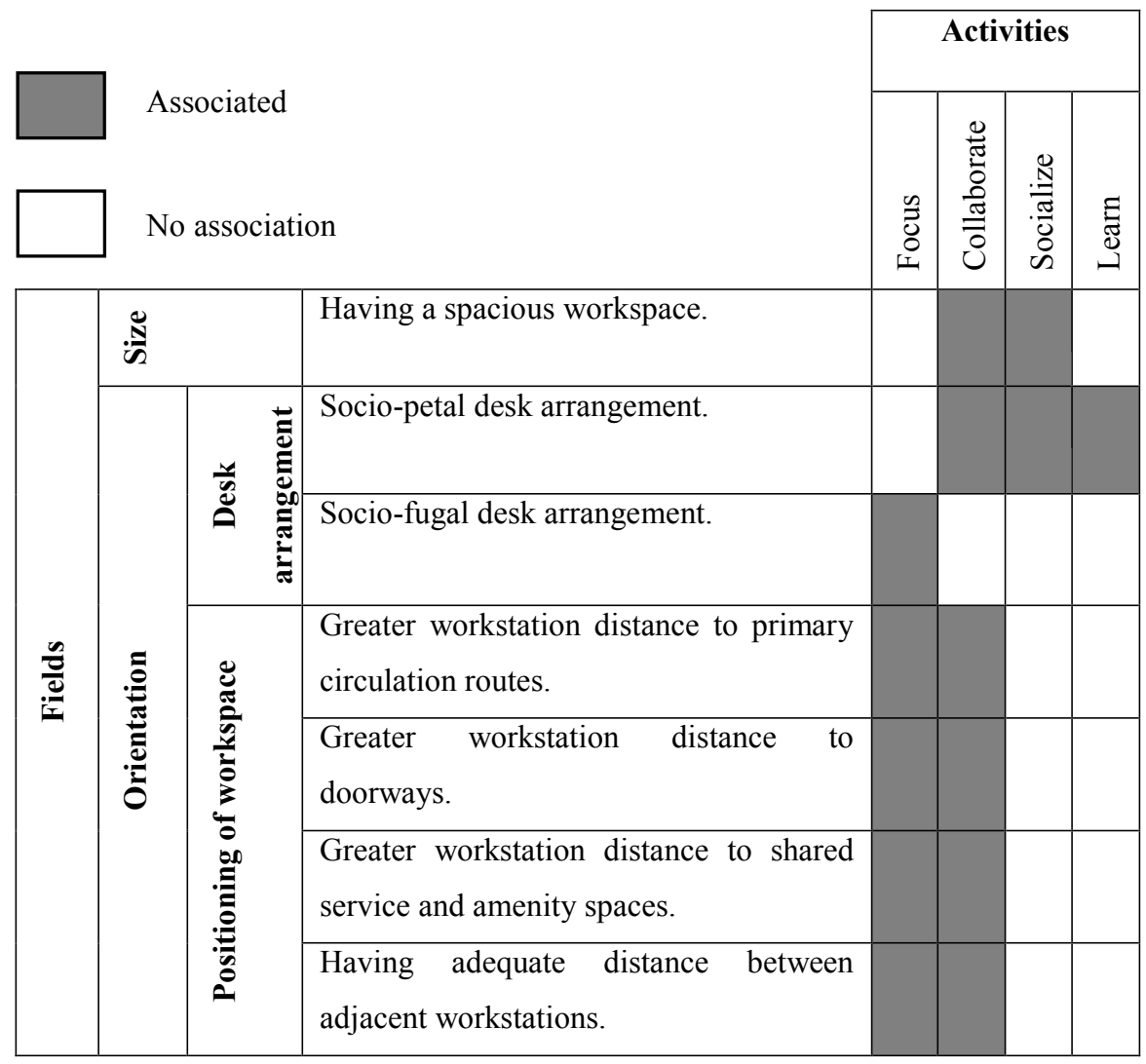

Table 4. Association between fields and users' activities

The information gathered in this study demonstrates that design elements comprising of barriers and fields could be incorporated in a practical way to afford desired behaviors in co-working spaces. Using a mix of free-standing barriers, an appropriate distance between workspaces and appropriate orientation or positioning of workspaces could afford users' needs to proficiently carry out the four work modes in co-working spaces.

\section{CONCLUSION}

Using an environment-behavior approach with ethnographic research techniques, this study investigated the relationship between spatial design and users' behaviors in co-working spaces. Specific spatial elements investigated in this study comprised of barriers and fields. The study has proved and demonstrated that spatial design significantly influences users' behaviors in coworking spaces. Since co-working is a new concept with limited empirical research, the data obtained from this study should be useful for architects, and facility managers when planning co-working spaces. Nonetheless, because the research was conducted through specific case studies, the findings might not be generalized to all co-working spaces. In future, a similar research could be carried out in co-working spaces from different countries and the outcomes of the research compared with each other in order to get more general findings. 


\section{REFERENCES}

[1] CHAN, J. K., BECKMAN, S. L., LAWRENCE, P. G., Workplace Design: A New Managerial Imperative, California Management Review, volume 49 (2007), no. 2, 6-22.

[2] SPINUZZI, C., Working Alone Together, Journal of Business and Technical Communication, volUME 26 (2012), no. 4, 399-441.

[3] https://hbr.org/2015/05/why-people-thrive-in-coworking-spaces

[4] ALLEN, T. J., HENN, G., The organization and architecture of innovation: Managing the flow of technology, Amsterdam Butterworth-Heinemann, 2007.

[5] DUFFY, F., POWELL, K., The New Office: Conran Octopus, 1997.

[6] HILLIER, B., Space Is the Machine: A Configurational Theory of Architecture: CreateSpace Independent Publishing Platform, 2015.

[7] https://www.gensler.com/uploads/document/126/file/2008_Gensler_Workplace_Workplace_Survey_US_09_30_2009.pdf

[8] ZEISEL, J., Inquiry by Design: Tools for Environment-Behaviour Research, Cambridge, UK: Cambridge University Press, 1984.

[9] BARKER, R. G., Ecological psychology : concepts and methods for studying the environment of human behavior, Stanford, California: Stanford University Press, 1968.

[10] GIBSON, J. J., The ecological approach to visual perception, Boston: Houghton Mifflin, 1979.

[11] HALL, E. T., The hidden dimension, Garden City, NY: Doubleday, 1966.

[12] LANG, J., Privacy, Territoriality and Personal Space - Proxemic Thoery. , Creating Architectural Theory: The role of the behavioral sciences in design, New York: Van Nostrand Reinhold, 1987. pp. 145-156.

[13] BRILL, M., MARGULIS, S. T., KONAR, E., BOSTI, Using office design to increase productivity, Buffalo, N.Y Workplace Design and Productivity, Inc., 1985.

[14] GOODRICH, R., Seven Office Evaluations, Environment and Behavior, volume 14 (1982), no. 3, 353-378.

[15] HEERWAGEN, J. H., KAMPSCHROER, K., POWELL, K. M., LOFTNESS, V., Collaborative knowledge work environments, Building Research \& Information, vol. 32 (2004), no. 6, 510-528.

[16] STUDER, R., The dynamics of behavior-contingent physical systems, Design Methods in Architecture, A. Ward and G. Broadbent, eds., London: Lund Humphries, 1969.

[17] MICHELSON, W. M., Behavioral research methods in environmental design: Dowden, Hutchinson \& Ross, 1975.

[18] CANTER, D. V., The psychology of place: Architectural Press, 1977.

[19] ITTELSON, W. H., LEANNE, G. R., HAROLD, M. P., The use of behavioral maps in environmental psychology, Environmental psychology: Man and his physical setting, H. M. Proshansky., W. H. Ittelson., L. G. Rivlin., eds., New York Holt, Rinehart and Winston, 1976.

\section{AcKNOWLEDGMENTS}

This article is adapted from the first author's master's thesis under the supervision of Asst. Prof. Dr. Sirimas Hengrasmee and Asst. Prof. Dr. Sant Chansomsak at Naresuan University, Thailand. Without their assistance and encouragement, this research would not have been accomplished. Finally, the authors would like to express their appreciation to the reviewers for their informative comments. 